\title{
Credit Risk Enhancement in a Network of Interdependent Firms*
}

\author{
Peter $\mathrm{Neu}^{1}$ and Reimer Kühn ${ }^{2}$ \\ ${ }^{1}$ Group Risk Control, Dresdner Bank AG, Jürgen-Ponto-Platz 1 \\ D-60301, Frankfurt, Germany \\ ${ }^{2}$ Department of Mathematics, King's College London \\ Strand, London WC2R 2LS, UK
}

First Version: August, 2003

This Version:

February 23, 2004

accepted for publication in Physica A

\begin{abstract}
We generalize existing structural models for credit risk to capture the impact of counterparty defaults on economic capital allocated to banks' loan portfolios. Exploring the analogy to a lattice gas model from physics, correlations between sequential defaults are modeled as due to functionally defined, heterogeneous couplings between mutually dependent counterparties. We show that - already for moderate micro-economic dependencies - counterparty risk results in a fattening of the tails in the portfolio loss distribution. In particular, for stronger mutually supportive relationship between the firms, collective phenomena such as bursts and avalanches of defaults can be observed in the model. In this context, traditional credit risk models are inadequate because they underestimate the required capital buffer. Our model setting is particularly applicable for doing stress analyses of credit risk in loan portfolios.
\end{abstract}

Key words: credit portfolio risk measurement, contagion, correlation, capital allocation

${ }^{*}$ Contacts: Peter Neu, +49 (69) 263 18582, Peter.Neu@Dresdner-Bank.com; Reimer Kühn, +44 (20) 7848 1035, kuehn@mth.kcl.ac.uk. The views presented in this paper are those of the authors and do not necessarily represent models or policies of Dresdner Bank AG. 


\section{Introduction}

In recent publications on credit risk modeling the impact of sequential defaults related to direct business relations between obligors has found much attention $[1,2,3,4,5,6,7,8]$. Research along this line, initiated by Davis and Lo [2], is motivated by attempts to explain the observed clustering of defaults around economic recessions [9], which cannot readily be accounted for by the standard approach for modeling correlations in either the reduced-form $[10,11,12]$, or the structural formulation $[13,14,15]$ of credit risk modeling. In these approaches default correlations are modeled by linking default intensities (reduced-form models) or asset returns (structural models) to macro-economical factors (e.g., interest rates, credit spreads, stock indices, economic growth). Since common factors weighted by some factor loadings influence the profitability of firms, equal-time default correlations emerge. Although $\mathrm{Yu}$ [16] has shown that the choice of the macro-economic factors can strongly influence the default correlation, research has recently also focused on explaining empirically observed correlations in terms of direct inter-firm relationships. Such relationships can arise from, e.g., (unsecured) borrowing and lending contracts in the interbank market or in economically bounded entities, such as conglomerates, supplier-manufacturer or parent-subsidiary structures. The results are functional inter-firm dependencies, which can initiate collective sequential defaults resulting in bursts and avalanches of defaults analogously to a first order phase transition in condensed matter systems [17]. ${ }^{1}$

The mechanism by which a default of a firm's counterpart might effect its own default probability was termed counterparty risk by Jarrow and $\mathrm{Yu}$ [3]. This mechanism adds further complexity to a credit risk model due to looping of default dependencies: essentially, every firm's survival at time $t$ depends on all other firms' survival at time $t^{\prime}<t$, which again depends on the original firm's survival at time $t^{\prime \prime}<t^{\prime}<t$. This kind of feedback generally prohibits straightforward exact analytical solutions. It is also the economic reason for cascading and clustering of defaults. To arrive at analytical, albeit approximate descriptions Jarrow and $\mathrm{Yu}$ eliminate looping defaults by considering an economy with two groups of firms: primary firms whose default depends only on marco-economic factors, and secondary firms whose default depends on marco-economic factors and defaults of primary firms.

The concept of functional dependence is not limited to credit portfolio perspectives. Sequential failure of processes constitute a main source for operational risk. A model exploring this notion in order to calculate the necessary capital buffer for operational risks was proposed by the authors in [19] and further elaborated by Leippold and Vanini [20].

The research initiated by Jarrow's and Yu's work followed the usual lines of either reduced-form $[3,4,5]$ or structural approaches $[6,7,8]$. Rogge and Schönbucher [4] apply a copula model in the reduced-form framework to counterparty risk. The choice of the copula function is determined by the distribution function of the common macro-economical factors. Their central result is that the conditional increase of the hazard rate can be expressed by the covariance of the corresponding common factors. This restricts their models to economies with symmetric counterparty risk, which is not the generic case. Frey and Backhaus [5] address the problem of looping default by a mean-field approximation in the reduced-form framework. This approximation, which is a common non-perturbative tool in statistical mechanics, allows them to go beyond the primary-secondary

\footnotetext{
${ }^{1}$ Similar positive feedback mechanisms are also believed to be responsible for the possible occurrence of catastrophic shifts in a variety of ecosystems [18].
} 
firm approximation of Jarrow and Yu. To apply mean-field theory the portfolio must be split into several homogeneous aggregates whose default statistics determines the default of an individual firm. Although this approximation might be important for portfolios with a large number of relatively homogeneous obligors, e.g., retail loan portfolios, it appears less well suited to model micro-economic business relationships in a corporate loan portfolio.

Counterparty risk has also been modeled in the structural formalism to credit risk. Giesecke and Weber $[6,7]$ apply a voter model with symmetrical nearest-neighbor couplings on a $d$-dimensional integer lattice $\mathbf{Z}^{d}$. Both, the regular geometric pattern of the functional inter-firm relations, and their uniform strength as well as their symmetry would have to be abandoned and replaced by less regular, heterogeneous variants in order to allow calibrating a model of this kind so as to a describe realistic networks of economic dependencies. Another point concerns the equilibrium assumption used in $[6,7]$, which is crucial for obtaining their main results, but which renders their analysis essentially static. Egloff et al. [8] also apply a structural model, with a linear coupling of asset returns between business counterparts. This linear feedback mechanism allows them to derive an analytical solution even for looping defaults. The fact that their model allows for heterogeneous and asymmetric dependencies opens ways for proper calibrations and thus creates considerable potential for practitioners. Model improvements can still be be made: it would, for instance, be more realistic to assume rating events (e.g., downgrade or default of a firm's counterpart) rather than a counterparty's asset returns (which are not directly observable in the market) to be the decisive quantities which influence a firm's own asset returns. This would imply a non-linear feedback mechanism between asset returns of related counterparts. Again, all results in [8] are obtained in the stationary equilibrium limit, although the authors also quote an autoregression formulation of their model. Finally, only the rating process is considered; the recovery process, which is important for applications to a bank's credit portfolio, is omitted.

The present paper attempts to provide and investigate some of the model improvements along these lines. Our analysis implies a general, non-geometric, heterogeneous and generally asymmetric form for the inter-firm dependencies. Moreover, as our prime focus here is to estimate the impact of counterparty risk in loan portfolios on capital allocation, we do not assume equilibrium dynamics here. This is an essential point, as risk capital, which is to be allocated as loss buffer in certain portfolios, is usually estimated on the basis of a healthy starting portfolio. Such a healthy portfolio constitutes a non-equilibrium initial condition. As, moreover, default probabilities $p$ are small (so that $1 / p \gg 1$ year), the portfolio will typically not reach an equilibrium state during the considered risk horizon for capital allocation (usually one year), which is why equilibrium results are strictly not applicable in this context.

As a result, analytical solutions are not easily available, though to a certain extent within reach of techniques developed in the statistical physics of disordered systems. Within the present paper we shall mainly resort to Monte Carlo simulations to elucidate the salient properties of our model.

Interest in capital allocation was renewed in the course of discussions in connection with the Basel II-process [21, 22, 23]. Following the functional approach proposed in [19], our goal is to formulate a generalization of the (structural) common CreditMetrics approach [24] that takes direct interactions between firms into account. The parameters of our model can be expressed analytically in terms of a priori and conditional default probabilities. The latter can be fixed by expert estimates, or from historical loss data. We will argue in particular that our model provides a consistent framework for stress testing of loan portfolios. 
The remainder of the paper is organized as follows. In Sect. 2 the standard approach to credit risk is extended for collective sequential default events based on heterogeneous, and generally non-symmetric functionally determined inter-firm dependencies. In Sec. 3 we explore the specific properties of our model and analyze cascading defaults using Monte Carlo simulations. In this section, we also comment on stress testing and discuss the issue of estimating the parameters for the model. Finally, Sect. 4 summarizes our results.

\section{Functional Approach to Counterparty Risk in Loan Portfolios}

We consider a simple two state model here for the $N$ firms in the loan portfolio of the bank, in which each firm has only been granted one single loan. Each firm can be either up and running or defaulted. For firm $i$ we designate these states in terms of an indicator variable $n_{i}$ as $n_{i}=0$ and $n_{i}=1$, respectively. In this setting only defaults trigger a credit deterioration of the loan portfolio. Influences of credit quality deterioration on the market value of the loan portfolio are, for simplicity, neglected, but they can easily be included in the scheme (e.g., by introducing multivalued as opposed to two valued indicator variables $n_{i}$ for the firms).

To motivate the dynamics of the functional approach, we assume that all firms need a certain amount of "orders," support, or cash inflow to avoid default during the time increment $t \rightarrow t+\Delta t$ within the risk horizon, $t \in[0, T$ ) (think of suppliers constructing small item to the product of the large-cap or subsidiaries receiving cheap funding from the mother firm, etc.).

We denote by $W_{i}(t)$ the total support received by firm $i$ at time $t$, and choose it to take the form

$$
W_{i}(t)=w_{i}^{(0)}-\sum_{j=1}^{N} w_{i j} n_{j}(t)+\eta_{i}(t)
$$

Economically, $W_{i}(t)$ can be considered as the "wealth" function of firm $i$ at time $t$. Alternatively, it can be interpreted as the asset return of the firm [13]. A positive wealth corresponds to an asset value which exceeds the debt of the firm. It is composed of (i) the average wealth $w_{i}^{(0)}$ that would be available in a fully operational network of firms, in which $n_{i}(t)=0$ for all $i$. (ii) This quantity can be diminished by defaults of firms $j$, whose business relation with $i$ are supportive $\left(w_{i j} \geq 0\right)$, so support from defaulted firms which normally feed orders into the firm in question would be missing; the converse - in case of competitors operating with similar products in the same market - could also occur; their relative 'coupling' $w_{i j}$ would be negative, and default of $j$ would increase the wealth of its competitor $i$, because $i$ would be able to take over a share of $j$ 's market. (iii) lastly, there are fluctuations about the average which we take to be (correlated) Gaussian white noise with-by proper renormalizing $w_{i}^{(0)}$ and $w_{i j}$-zero mean and unit variance.

Firm $i$ will default in the next time instant $t+\Delta t$, if its wealth becomes negative, $W_{i}(t)<0$, thus

$$
n_{i}(t+\Delta t)=\Theta\left(-w_{i}^{(0)}+\sum_{j} w_{i j} n_{j}(t)-\eta_{i}(t)\right)
$$


where $\Theta$ denotes the step-function: $\Theta(x)=1$ for $x \geq 0$ and $\Theta(x)=0$ otherwise. The decisive point about this dynamical rule is that defaulted firms can (and generally will) change the probability of default for other firms in the future, and it will increase their probability of default if their economic relation is mutually supportive $\left(w_{i j}>0\right)$.

At this point it must be noticed that there is an important difference in the dynamics as compared to that employed in the formally rather similar case of operational risks [19]. Namely, one usually assumes that a defaulted firm will not recover during the risk horizon $T$; in a technical sense, therefore, $n_{i}=1$ is an absorbing state, so the dynamical rule (2) ought to be supplemented with

$$
n_{i}(t+\Delta t)=1, \quad \text { if } \quad n_{i}(t)=1 .
$$

A defaulting firm will generate losses for the bank lending to it. Hence the losses $L_{i}$ incurred due to firm $i$ have to be updated according to

$$
L_{i}(t+\Delta t)=L_{i}(t)+n_{i}(t+\Delta t) E_{i}(t+\Delta t) L_{D}^{(i)}(t+\Delta t),
$$

where $E_{i}$ denotes the exposure at default and $L_{D}^{(i)}=1-R^{(i)}$ the loss given default, $R^{(i)}$ being the recovery rate. $E_{i}$ is the amount that will be lost due to outstanding transactions with the $i^{\text {th }}$ firm, not adjusted for any future recovery and incomes during the bankruptcy process. $L_{D}^{(i)}$ is this adjustment factor such that $E_{i} \times L_{D}^{(i)}$ is the loss severity. Generally, $E_{i}$ and $L_{D}^{(i)}$ are taken to be time-dependent random variables, e.g., think of credit lines where the future draws are not known a-priori. It is understood that (4) applies at default only. It is worth pointing out here that the (stochastic) model of incurred losses for a given default (i.e. the model of the recovery process) is independent of the model assumed for the underlying dynamics of firm.

We will be following widespread practice (e.g. [15, 24, 25]) to model common systematic, albeit fluctuating risk factors, which represent the industry sector, region, or country specific influence on the economy, by taking the noise contribution $\eta_{i}(t)$ in (2) to be correlated for different $i$. By this device, one can model equal-time cross correlations between firm defaults. Specifically, we take

$$
\eta_{i}(t)=\sum_{k=1}^{K} \beta_{i k} Y_{k}(t)+\xi_{i} \epsilon_{i}(t)
$$

where

$$
\xi_{i}=\left(1-\sum_{k=1}^{K} \beta_{i k}^{2}\right)^{1 / 2} .
$$

Thus, the $Y_{k}(t) \sim \mathcal{N}(0,1)$ represent the common risk factors, whereas the $\epsilon_{i}(t) \sim \mathcal{N}(0,1)$ are firm-specific, idiosyncratic risk factors describing fluctuations in the micro-economical state of the firms. All firms in the same risk sector $k$ are subject to the same systematic factor, $Y_{k}(t)$. However, each of them has an individual component through the idiosyncratic term, $\epsilon_{i}(t)$. The weights $\beta_{i}=\left(\beta_{i 1}, \ldots, \beta_{i K}\right)$ stand for the sensitivity of the wealth of firm $i$ to changes in $k^{\text {th }}$ risk sector. For credit risk they are always positive or zero, $\beta_{i k} \geq 0$. Without loss of generality we assume the risk sector to be orthogonal, i.e., the $Y_{k}(t)$ to have zero correlation. ${ }^{2}$ Equal-time asset correlations

\footnotetext{
${ }^{2}$ This can always be assured by a simple rotation of "economic" risk sectors. The $Y_{k}(t)$ would then be linear combinations of factors associated to these "economic" risk sectors.
} 
are then given by $\rho_{i j}=\sum_{k} \beta_{i k} \beta_{j k}$ for $i \neq j$. It is understood that the dynamics of the common economic factors $Y_{k}(t)$ is very slow on the time scale set by $\Delta t$.

The microscopic parameters $w_{i}^{(0)}$ and $w_{i j}$ describing the dynamics of our model can be related to statistical properties of default events as follows. Denoting by $p_{i, \Delta t}$ the unconditional probability for firm $i$ defaulting within the time increment $t \rightarrow t+\Delta t$, given that no other firm has defaulted at time $t$, i.e., $n_{j}(t)=0$ for all $j$,

$$
p_{i, \Delta t} \equiv \operatorname{Prob}\left(n_{i}(t+\Delta t)=1 \mid\left\{n_{j}(t)=0\right\}\right),
$$

one obtains $p_{i, \Delta t}$ by integrating over the $\mathcal{N}(0,1)$ noise $\eta_{i}$ in $(2)$, giving

$$
p_{i, \Delta t}=\Phi\left(-w_{i}^{(0)}\right) .
$$

Here, $\Phi$ denotes the cumulative normal distribution $\Phi(x)=\frac{1}{2}(1+\operatorname{erf}(x / \sqrt{2}))$. Inverting (8) one obtains

$$
w_{i}^{(0)}=-\Phi^{-1}\left(p_{i, \Delta t}\right) .
$$

Similarly,

$$
p_{i j, \Delta t} \equiv \operatorname{Prob}\left(n_{i}(t+\Delta t)=1 \mid n_{j}(t)=1,\left\{n_{k}(t)=0, k \neq j\right\}\right),
$$

i.e., the probability of firm $i$ defaulting within the time increment $t \rightarrow t+\Delta t$ conditioned on firm $j$ being defaulted at $t$ is obtained by integrating over the $\mathcal{N}(0,1)$-noise $\eta_{i}$ in (2) the same manner, the only difference being in the configuration $n(t)=\left\{n_{i}(t)\right\}$ assumed at time $t$. Inverting and using the result (9), one obtains

$$
w_{i j}=\Phi^{-1}\left(p_{i j, \Delta t}\right)-\Phi^{-1}\left(p_{i, \Delta t}\right) .
$$

Note that $p_{i j, \Delta t}$ are stationary transition rates of the Markov process in terms of which the dynamics of our model is defined; they can be interpreted as a non-equal time cross-correlation of obligor default within the time increment $\Delta t$.

Finally, in order to investigate the dynamics in detail, e.g., by setting up a Monte Carlo simulation, the conditional probability for default of firm $i$, during the time increment $t \rightarrow t+\Delta t, p_{i, \Delta t}(n, Y)$, given a configuration $n(t)=\left\{n_{i}(t)\right\}$ of defaulted firms and a realization $Y(t)=\left\{Y_{k}(t)\right\}$ of common factors at time $t$ is needed. It is obtained by integrating over the distribution of idiosyncratic noises, $\epsilon_{i}$, in such a configuration. Combining (2) and (5), one obtains

$$
\begin{aligned}
p_{i, \Delta t}(n, Y) & \equiv \operatorname{Prob}\left(n_{i}(t+\Delta t)=1 \mid n(t), Y(t)\right) \\
& =\Phi\left(\frac{\Phi^{-1}\left(p_{i, \Delta t}\right)+\sum_{j} w_{i j} n_{j}(t)-\sum_{k} \beta_{i k} Y_{k}(t)}{\sqrt{1-\sum_{k} \beta_{i k}^{2}}}\right) .
\end{aligned}
$$

Note that up to the functional term " $\sum_{j} w_{i j} n_{j}(t)$ " this approach corresponds to the standard approach, see e.g. [15, 24], for credit risk modeling, which in the one-factor version and in the limit of an infinitely fine-grained portfolio is also the basis for Basel II [21, 22, 23].

Equation (12) has a very intuitive economic interpretation. Defaulted firms which were in a mutually supportive economic relation with the firm under consideration $\left(w_{i j}>0\right)$, or negative common factors increase its conditional default probability, and, henceforth, result in enhanced economic 
distress. Conversely, defaulting firms which were in a mutually competitive economic relation with the firm under consideration $\left(w_{i j}<0\right)$, or positive common factors would decrease its conditional default probability, and, henceforth, result in reduced economic distress.

Let us emphasize one further important consequence of our way of thinking about the dynamics underlying credit risk. It is related to the observation that the economic network of dependent firms is usually (much) larger than the set of firms in a bank's lending portfolio. This aspect is only partially taken into account by the introduction of the set of common risk factors $\left\{Y_{k}\right\}$ in (5), namely only to the extent that adverse effects on the bank's lending portfolio through defaults of individual, identifiable firms outside the bank's own portfolio can be neglected. This is quite obviously not always appropriate. Within the present approach these considerations can easily be taken into account by looking at the dynamics of larger networks of interacting firms and setting $E_{i}=0$ for the firms that are not in the bank's portfolio.

\section{Properties of the Model}

For a general heterogeneous network of firms the dynamics (1), (2), and (12) cannot be solved analytically. To study the salient properties of our model we shall, therefore, resort to Monte Carlo simulations. Note that, within the present paper, we do not attempt to simulate networks with parameters derived from a realistic economic network, but rather simulate the heterogeneity of realistic economic networks by using a fixed random setting for the dependencies in the economic network of interacting firms. That is, we shall be using fixed (quenched) random unconditional and conditional default probabilities for the firms, and we shall in the present paper also not consider the case where the economic network is larger than the network of firms in the bank's lending portfolio. Moreover, to emphasize the effects of interaction-induced enhancement of credit risk, we shall in the present setup consider a network containing only mutually supportive firms.

It is clear that none of the specifications chosen constitute restrictions of principle. Some could be read as attempts at generality; the last is merely meant to illustrate more succinctly the collective effects that may occur in such systems.

We will show that, with economic stress percolating through the portfolio of mutually dependent firms, cascading defaults can fatten the tail of the loss distribution, and even render it bimodal in the limit of strong dependencies.

\subsection{Setting up the Monte Carlo Simulation}

To simplify matters further we restrict ourselves to having just a single common macro-economical factor so that

$$
\eta_{i}(t)=\sqrt{\rho} Y(t)+\sqrt{1-\rho} \epsilon_{i}(t)
$$

with correlation coefficient $\rho$. Furthermore, we consider a homogeneous portfolio in which we chose $E_{i}$ to be the same, and equal to 1 , for each firm. $L_{D}$ is sampled from a beta-distribution upon each default.

Eq. (12) constitutes the basis of the Monte Carlo algorithm. The probabilistic dynamics is carried out in parallel. We take the time increment $\Delta t$ to represent a single day. The common economic 
factor is assumed to vary more slowly than on a day-to-day basis. Within the present simulations we choose to update it just once during a year from a zero-mean unit-variance Gaussian. One year is also a common choice for the value of the risk horizon $T$ in the problem of capital allocation for covering credit risk. Indeed, for the question of estimating the annual loss distribution of a loan portfolio, which is the question we decided to address with the simulations presented below, the question of the dynamics of the common factor is not of prime importance, as long as it is agreed that the time-scale of its variations is of the order of a year: the loss distribution is in any case calculated for portfolios starting in a healthy state at the beginning of the risk horizon, i.e., in a non-equilibrium initial condition without defaults. Other ways of setting up a simulation are of course conceivable, when other questions are being addressed.

For fixed $Y$, Eq. (12) is sampled for a risk horizon of $T=365$ days, starting with $n_{i}(0)=0$ for all $i$. In case of a default under the given scenario - a default occurs in the simulation, if a $[0,1]$-distributed random variable is smaller than the conditional default probability, Eq. (12) - a random variable for $L_{D}$ is drawn from a beta-distribution for constant $E$. Repeating this for many years with $Y$ annually chosen according to its distribution, a histogram of the annually realized portfolio losses is eventually obtained. The economic capital (EC) is read off from the $q$-quantile of this histogram,

$$
L_{q}(T)=\inf \left\{L(T): \operatorname{Prob}\left(L(T) \leq L_{q}(T)\right) \geq q\right\}
$$

in excess of the expected loss, $\langle L(T)\rangle,(\langle\cdot\rangle$ denoting the average over the loss distribution function)

$$
\mathrm{EC}(T)=L_{q}(T)-\langle L(T)\rangle
$$

Economic capital is the industry standard for the amount of equity capital required to buffer unexpected losses (risk) at a certain confidence level $q$. Commonly, the confidence level is determined from the external (S\&P or Moody's) target rating of the bank such that $q=1-p_{1 Y}$ (target rating).

To set up our random realization of a network of interacting firms, we choose fixed unconditional default probabilities $p_{i, \Delta t}$ for each firm, taken to be homogeneously distributed in some interval $\left[0, p^{\max }\right]$. The transition probabilities $p_{i j, \Delta t}$ are also fixed, and randomly chosen as

$$
p_{i j, \Delta t}=p_{i, \Delta t}\left(1+\varepsilon_{i j}\right)
$$

with $\varepsilon_{i j}$ taken to be homogeneously distributed in the interval $\left[0, \varepsilon^{\max }\right]$. This fixes the ratio $\left(p_{i j, \Delta t} / p_{i, \Delta t}\right)^{\max }=1+\varepsilon^{\max }$. For a specific economic network, the parameters $p_{i, \Delta t}$ and $\varepsilon_{i j}$ would have to be determined from from expert assessment, on the basis of historical data, or otherwise (we shall return to this issue in greater detail below). The random specifications made here have no particular claim on realism in economic detail; they are just meant to be reasonable, and able to elucidate the main principles at work in an economic network of mutually supportive firms.

\subsection{Results}

The values of $p^{\max }$ and $\varepsilon^{\max }$, and of the correlation coefficient $\rho$ are the control parameters of our system. Within the present study, simulations were carried out for a network of $N=100$ interacting firms, and $p^{\max }=5 /(365 \times N)$, that is an unconditional default probability homogeneously distributed in the range between 0 and 5\% was chosen for the data presented in Figs. 1 and 2 . 


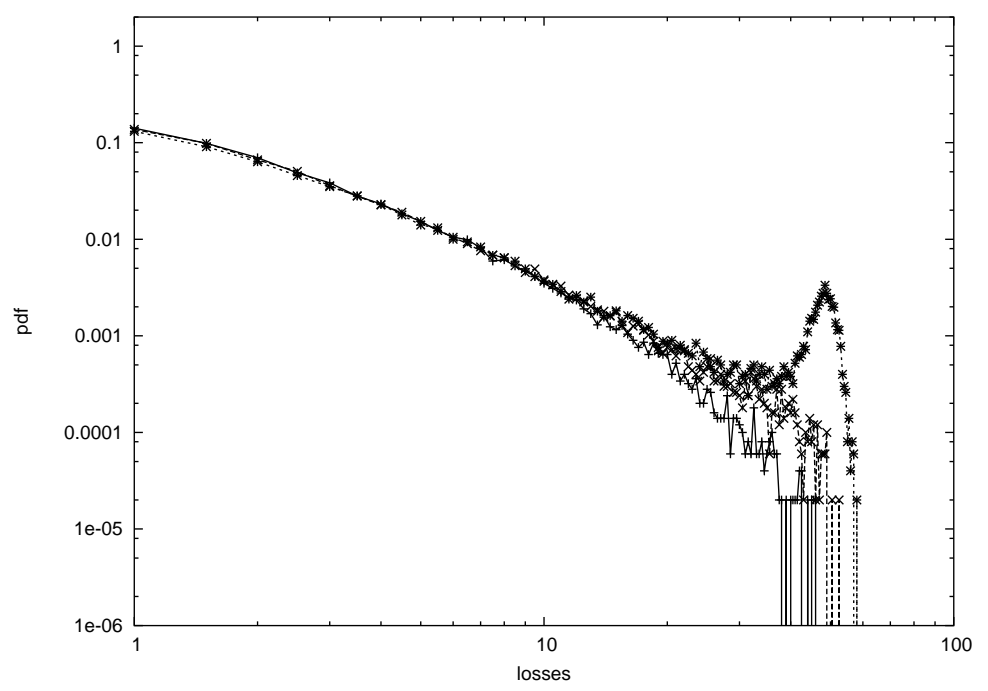

Figure 1: Probability density function of the losses for a fully connected network of $N=100$ firms, $p^{\max }=5 /(365 \times N), \rho=15 \%$, and $T=365 \times \Delta t$. From left to right the curves correspond to the cases of non-interacting firms (crosses), the case $\left(p_{i j, \Delta t} / p_{i, \Delta t}\right)^{\max } \equiv 1+\varepsilon^{\max }=1.04$ (diagonal crosses), and $\left(p_{i j, \Delta t} / p_{i, \Delta t}\right)^{\max } \equiv 1+\varepsilon^{\max }=1.16$ (asterisks), a case where the entire network of loans may collapse.

The correlation coefficient between the fluctuating forces originating from the common factor $Y$ was taken to be $\rho=15 \%$, and the parameters of the beta-distribution used for sampling the $L_{D}$ 's are $a=b=3 / 2$, giving a semicircular law for the corresponding probability density function with mean loss rate $\overline{L_{D}}=50 \%$ and $L_{D}$-volatility $\sigma_{L_{D}}=25 \%$.

If the unconditional default probabilities $p_{i, \Delta t}$ and the transition probabilities $p_{i j, \Delta t}$ are small enough to allow for a stable economy, one finds that the loss distribution is always unimodal with frequent small losses and a few extreme losses, which together with the randomness of the loss severity are responsible for tails in the loss distribution. With increasing $\left(p_{i j, \Delta t} / p_{i, \Delta t}\right)^{\max }$, counterparty risk in the portfolio and with it the likelihood of larger losses increases. If the ratio $\left(p_{i j, \Delta t} / p_{i, \Delta t}\right)^{\max }$ increases beyond a critical value, collective losses, or crashes, in the form of bursts or avalanches of default events, spreading through the whole economy, will occur-the economic network of interacting firms will become collectively unstable. The avalanches of default events are completely analogous to bubble nucleation associated with first order phase transitions, and will cause the loss distribution to become bimodal with a marked peak at very high losses. Fig. 1 documents this scenario by comparing the loss distribution one would expect for non-interacting firms with two cases where the ratio $\left(p_{i j, \Delta t} / p_{i, \Delta t}\right)^{\max }$ is increased to 1.04 and 1.16 , respectively.

Note that even in the case of weak inter-firm dependencies, i.e., only $4 \%$ enhancement of the default probability, the loss distribution already extends to significantly larger values: the $99.5 \%$ quantile increases by approximately $40 \%$ (from $L_{99.5} \simeq 17.0$ to $L_{99.5} \simeq 24.0$ ), while the increase of the expected loss is by approximately $15 \%$, and thus much less pronounced. The reason for the rather drastic effect of inter-firm dependencies in the present setup is the high connectivity of the 
network, where all $N=100$ firms are chosen to interact with each other.

Indeed for the present fully connected network the larger of the two ratios, $\left(p_{i j, \Delta t} / p_{i, \Delta t}\right)^{\max }=1.16$, is large enough for collective instabilities of the network of interacting firms to occur, causing the loss distribution to become bimodal with a marked peak at very high losses, as shown in Fig. 1, and leading to an increase of of the $99.5 \%$ quantile of the loss distribution relative to the non-interacting case by approximately $300 \%$ !

Fig. 2 shows the behavior of the economic capital as function of the control parameter $\left(p_{i j, \Delta t} / p_{i, \Delta t}\right)^{\max }=1+\varepsilon^{\max }$, normalized with respect to the case of non-interacting firms. Note the relative sharp rise at intermediate values of $1+\varepsilon^{\max }$ for the fully connected case. This sharp rise is a trace of an underlying first order phase transition. In our simulations, that transition is 'smeared.' The reason here is not so much that we are dealing with a relatively small network, but also, because we are simulating the system over a finite time-window of the length of the risk horizon $T$, starting with a healthy portfolio. Hence, we consider a finite time window of a nonequilibrium dynamics. In our simulation we are therefore always only seeing the first stages of a bubble nucleation associated with an underlying phase transition.

In the limit of large networks and large time-horizon $T$ such that equilibrium conditions apply, the economic capital would, indeed, develop a true jump discontinuity, as $1+\varepsilon^{\max }$ is increased beyond a given critical parameter. We have not attempted to precisely locating the transition point via a finite size scaling analysis.

Clearly, the rise is less dramatic for lower, and more realistic connectivities of the network as shown in Fig. 2. However, for the lower connectivity, too, there would be a critical value of the control parameter $\left(p_{i j, \Delta t} / p_{i, \Delta t}\right)^{\max }=1+\varepsilon^{\max }$ at which a sharp rise of the economic capital as a function of this parameter would be observable and develop into a true jump discontinuity in the limit of large system sizes and long risk horizons.

The second panel in Fig. 2 compares the result obtained for the case of $20 \%$ connectivity with the result for a collection of non-interacting firms with unconditional default probabilities $p_{i, \Delta t}$ uniformly sampled from the interval $\left[0, p^{\max } \times\left(1+0.2 \varepsilon^{\max }\right)\right]$. It demonstrates that the effect of counterparty risk on economic capital cannot be covered by solely renormalizing unconditional default probabilities, which is a common pragmatic approach in the banking industry.

Our results do not contradict arguments made in $[3,8]$ according to which effects of counterparty risk will disappear in large well-diversified portfolios. Quite to the contrary, our model is fully compatible with that reasoning, the key point being that large bond portfolios such as the Moody's or S\&P bond universe do not only contain bonds of mutually supportive firms (with $w_{i j}>0$ ) but also bonds of mutually competitive firms (with $w_{i j}<0$ ). Hence, counterparty risk in such portfolios is (partially) diversified away exactly because mutually supportive and competitive interfirm dependencies are present. This has an important implication for the credit process: banks can naturally hedge counterparty risk by granting loans to competing firms.

\section{3 $\quad$ Stress Testing}

Stress testing is an important part of modern risk management. Whereas common Value-at-Risk measures refer to a worst case loss under normal market conditions, stress tests constitute the complementary part in the analysis of the risk profile by considering specific crisis scenarios. 

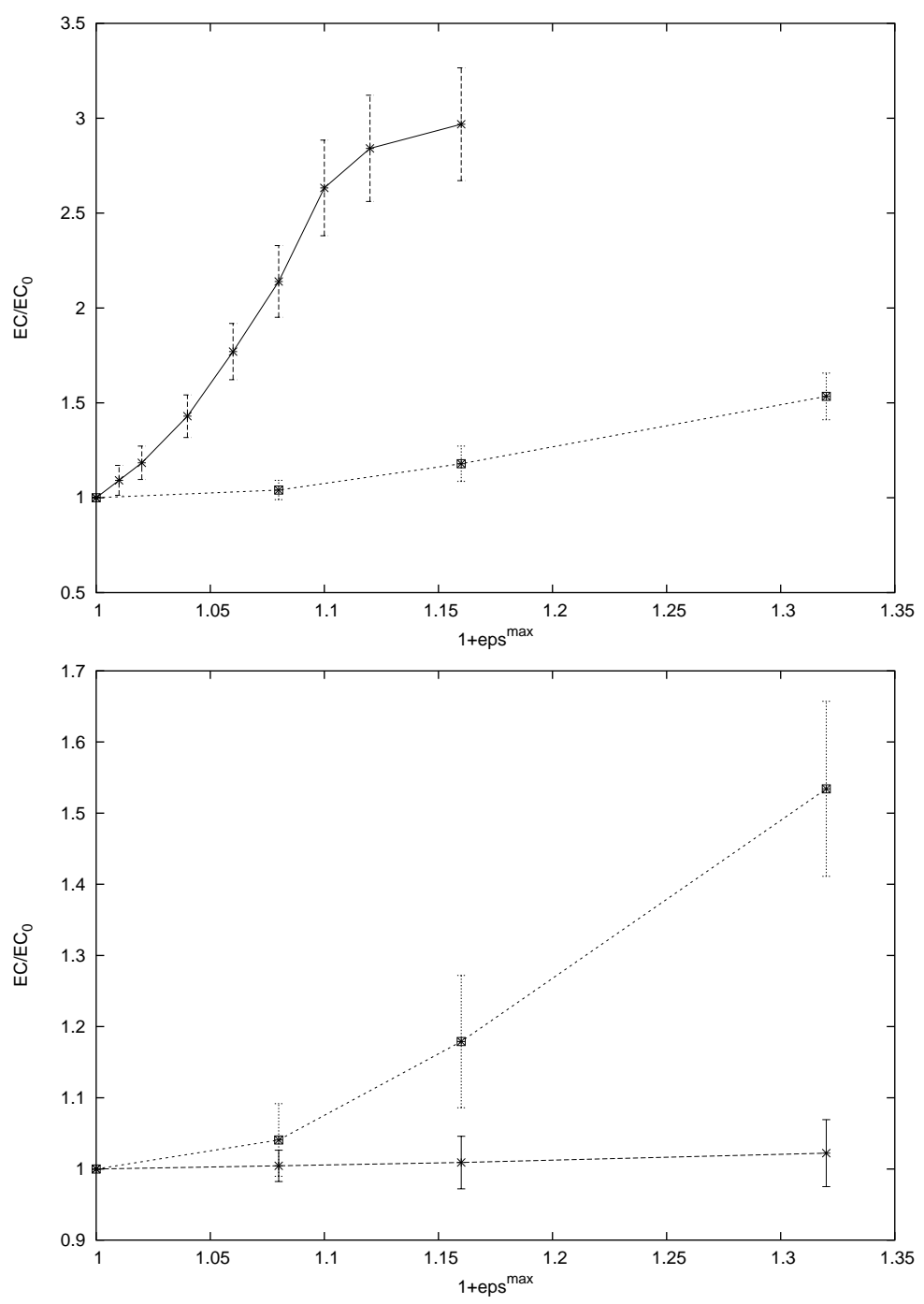

Figure 2: Economic capital EC $=L_{99.5}-\langle L(T)\rangle$ as a function of the control parameter, $\left(p_{i j, \Delta t} / p_{i, \Delta t}\right)^{\max }=1+\varepsilon^{\max }$ relative to its value $\mathrm{EC}_{0}$ for non-interacting firms for $N=100$ firms, $p^{\max }=5 /(365 \times N), \rho=15 \%$, and $T=365 \times \Delta t$. In the first panel, the upper curve gives the behavior for a fully connected network, the lower curve for a network of the same size, but each firm interacting only with a randomly chosen subset of $20 \%$ of the other firms in the net. The second panel compares the result obtained for the case of $20 \%$ connectivity with the result for a collection of non-interacting firms with unconditional default probabilities $p_{i, \Delta t}$ uniformly sampled from the interval $\left[0, p^{\max } \times\left(1+0.2 \varepsilon^{\max }\right)\right]$. 
There is no generally agreed approach for stress testing of loan portfolios. In the standard approach without the " $\sum_{j} w_{i j} n_{j}(t)$ "-term in (12) it has been suggested to do stress testing by setting all factor loadings $\beta_{i k}$ close to unity. Although this device increases the economic capital, such an approach can be criticized. It addresses only systematic stress events, like recessions in industry or country sectors. Specific stress events like the default of a large borrower are not considered. In our approach a much more controlled and bank-specific stress test of a loan portfolio is possible by setting specific firms into the default state - simply by turning specific $n_{j}(t) \equiv 1$ in the " $\sum_{j} w_{i j} n_{j}(t)$ "-term - and analyzing the impact on the loss distribution.

One of the most critical lessons for Risk Control from our analysis is the possible metastability of networks of interdependent firms: the bank would not necessarily realize the potential of big losses due to bursts and avalanches of obligor defaults, as there are no detectable precursors to such events for large portfolios. A basically unchanged loan portfolio could collapse and cause significant losses, either due to external strain or rare fluctuations of internal dynamics. The likelihood of such a collapse of the loan portfolio would, however, be revealed by our analysis.

\subsection{Calibration of Model Parameters}

The present model requires the specification of unconditional default probabilities $p_{i, \Delta t}$, factor loading $\beta_{i k}$, and of conditional default probabilities $p_{i j, \Delta t}$. The procedure to calibrate unconditional default probabilities and factor loadings from empirical default data of diversified portfolios, e.g., the S\&P or Moody's bond universe, is known to practitioners [25], and succinctly described, e.g., by Egloff et al. [8].

In the present section we discuss ways to estimate unconditional, $p_{i, \Delta t}$, and conditional default probabilities $p_{i j, \Delta t}$ for ordinary loan portfolios of banks, bearing in mind that these 'bare' quantities may be difficult to measure directly in these portfolios. They must be extracted from default statistics that is already renormalized through interactions, as it originates in a world of interdependent firms. It is clear that such estimates can only be obtained by refining current rating procedures.

In a conventional rating process obligors are assigned to a rating grade, e.g., $\mathrm{A}-, \mathrm{BBB}+$, or BBB, depending on their score $Z$ in a rating system. The score is given as some (non-) linear function $f$ of individual rating factors $X_{1}, \ldots, X_{n}, Z=f\left(a_{1} X_{1}+a_{2} X_{2}+\ldots a_{n} X_{n}\right)$, with factor loadings $a_{1}, \ldots, a_{n}$. In a rating calibration the function $f$ and the factor loadings $a_{\alpha}$ are optimized over the ensemble in such a way that the statistics of predicted defaults matches that in a historical test sample as closely as possible. The bucketing in rating grades is done such that the associated average default probability matches the portfolio number of defaults and the entire buckets cover the default distribution of the portfolio. Default probabilities are estimated ex post from the number of actual defaults per rating grade.

In a reasonable rating system the rating factors typically include balance sheet key figures (equity/debt capital ratio, legal structure, profit, sales, cost-income ratio, etc.), management factors (good/bad management, strategic planning, controlling processes, disclosure, etc.), market segment figures (economic cycle, segment economic indicators, etc.) and market position figures (comparison to competitors, offered products, customer/supplier structure, etc.). Hence, due to the last group of rating factors, such a rating system does take economic inter-firm dependencies into account. As 
a result, corporate dependencies do in the rating process conventionally result in assigning obligors to a lower, less qualified rating grade. As a consequence, the calibration of rating grades will lump both, a-priori, and conditional default probabilities into a single assigned default probability. In a certain sense, therefore, such a conventional rating procedure performs an implicit type of renormalization of the unconditional default probability in such a way that detailed information about economic dependencies, which as argued above would be very useful for specific stress testing, is virtually lost.

From a fundamental point of view, it seems very difficult to disentangle unconditional and conditional default probabilities from available historical data. In practice, estimating these parameters with a degree of (im)precision similar to that embodied in the implicit renormalization scheme followed by the conventional combination of rating scheme and calibration of rating grades would, however, not seem entirely unrealistic. For practitioners the focus must clearly be on using simplest models with smallest number of extra parameter that will capture counterparty risk in a credible way. Even if statistical estimates are hard to come by, plausible ranges of parameters can be derived on intuitive grounds. Analyses using plausible values give bounds on capital charges and are certainly better than ignoring counterparty risk altogether. According to this, a representation such as that following Eq. (16) above, which estimates a suitable range for the conditional default probabilities based on an expert assessment for $\varepsilon^{\max }$, could constitute a reasonable first pragmatic solution to the calibration problem.

Any way of going beyond such a first approximate solution of the calibration problem would have to take conventional rating procedures as a starting point, but would require refining them in suitable ways. Here, we just hint at one possible way of attacking this problem by formulating a bootstrap algorithm. It is based on the observation that a calibrated rating procedure can be thought of as providing a function $\mathcal{P}$ that maps scores $Z$ on interaction-renormalized default probabilities (within a time increment $\Delta t$ ); before binning into discrete rating grades, this function will be smooth and monotone. Given the score $Z_{i}$ of firm $i$, one may compute a related 'bare score' $Z_{i}^{0}$ for the same firm by ignoring the contributions of rating factors describing economic inter-firm dependencies (by resetting the corresponding factor loadings to zero). To the extent that the remaining rating factors are independent of those characterizing economic inter-firm dependencies, the default probability computed for the bare score $Z_{i}^{0}$ may then be taken to provide an estimate of the unconditional default probability $p_{i, \Delta t} \simeq \mathcal{P}\left(Z_{i}^{0}\right)$ needed within the present approach.

The values of the $\varepsilon_{i j}$ introduced in (16) could be fixed by asking experts to estimate the relative values of the transition probabilities, i.e., ratios of the form

$$
R_{j k}^{(i)}=\frac{p_{i j, \Delta t}}{p_{i k, \Delta t}}=\frac{1+\varepsilon_{i j}}{1+\varepsilon_{i k}} .
$$

While considering a single such ratio is insufficient to fix the values of the two $\varepsilon$ 's involved, the estimate of three such ratios (with a common $i$ ) involving a closed loop $(j, k, \ell)-R_{j k}^{(i)}, R_{k \ell}^{(i)}$ and $R_{\ell j}^{(i)}$-are sufficient to fix the three $\varepsilon$ 's involved. Further iterative improvement and consistency checks, and thus systematic bootstrapping is possible by considering longer loops. Together with the $p_{i, \Delta t}$, this allows to compute the conditional default probabilities $p_{i j, \Delta t}$, hence, the couplings used in our model. If need be, additional refinement of model parameters could be obtained using so called Reverse Monte Carlo methods (reviewed, e.g., in [26]). 
The suggested refinement of the rating process is clearly very work-intensive and requires high standards on the credit process of a bank. It is also clear that a proper implementation of such a calibration procedure constitutes a major research project in its own right.

Although it may be difficult to determine model parameters with high accuracy within such a procedure, the approach may nevertheless be viable for two reasons. One is that the renormalization effect on the default probabilities for weak dependencies is expected to be small anyway, and in any case not much larger than the error margins of default probabilities determined within a conventional rating procedure. Hence, no substantial additional source of errors is likely to be introduced in being a bit more explicit about the renormalization effect. More importantly, the other reason is that macroscopic properties of interacting systems of the type considered here are quite often fairly insensitive to variations at the microscopic level. Only statistical properties of the model parameters - not individual parameters themselves - must be captured with reasonable accuracy in order to allow reliable predictions about its global statistical properties such as, indeed, the properties of loss distributions.

\section{Conclusion}

To summarize, we have introduced and analyzed a framework that allows to estimate the effects of functional inter-firm dependencies within a collection of firms in a bank's lending portfolio on credit risk. We have demonstrated that the model parameters can be expressed in terms of a-priori and conditional default probabilities. We have indicated ways in which these 'bare' default probabilities that would have to be used in the model could be estimated by taking a closer look at the rating process.

The analysis in this paper has shown that the standard models for credit risk, which are based on equal-time correlations to common macro-economical environment, could significantly underestimate the losses of credit portfolios. Corporate dependency results in counterparty risk and can significantly amplify portfolio losses. Our main point was that functional correlations between mutually supportive firms give rise to non-trivial temporal correlations, which could eventually lead to the collective occurrence of losses in form of bursts, avalanches or crashes.

Conversely, by following the same kind of reasoning one easily convinces oneself that the mechanisms responsible for a possible amplification of portfolio losses in a network of mutually supportive and thus mutually dependent firms can be counter-balanced by setting up a loan portfolio in such a way that it includes a balance of mutually supportive and mutually competitive firms. This would create functionally induced "anti-correlation effects," which work to reduce the economic capital of a lending portfolio. This aspect gains particular importance in secondary loan trading markets and for loan portfolio management. Note that very much the same set of ideas are well known in the context of portfolio optimization with regard to market risk.

In any case there are important implications for the management of credit risk in loan portfolios: our analysis calls for much higher qualitative and quantitative standards in the rating process. It demonstrates that stress analyes are required to assess possible metastabilities of the loan portfolio. Indeed, an important property of our model is its appropriateness for stress analysis and the possibility to describe metastable networks of corporate dependent firms. Credit risk migration from external strain or internal defaults can be modeled in a conceptually well-defined framework, 
and crashes, bursts or avalanches of defaults are - in contrast to the traditional models - part of the default dynamics.

Another point worth returning to is connected with credit risk migration from outside the bank's own loan portfolio. An event, which could trigger major losses for the bank in question through collective effects, would be the default of a major player which - while not contributing to the bank's own loan portfolio - may nevertheless be economically well connected to the network of firms that constitute the bank's portfolio. As mentioned earlier, our analysis of credit risk migration may well be set up in ways that take the possibility of such 'Trojan Horse' effects properly into account. The case of the retail portfolio of a small local bank providing mortgages to housekeepers predominantly working for the same large employer in the area can provide an example for such considerations. In such a case, the renormalization of default probabilities due to interactions in setting up the model may safely be ignored, whereas the significant enhancement of default probabilities due to a possible default of the large local employer creates the major source of risk for the bank.

In conclusion, if counterparty risk in banks' loan portfolios is ignored, the calculated economic capital may well understate the appropriate risk buffer. Proper stress and risk migration analysis, akin to those outlined in this paper, could turn out an important tool for assessing the resilience of a bank's loan portfolio against dangers of catastrophic collective losses.

Acknowledgments. We would like to thank Frank A. Breitling from Dresdner Bank AG for valuable discussions and practical input. 


\section{References}

[1] Moody's Investment Services, The binominal expansion technique, URL: www.moodys.com (1997).

[2] M. Davis and V. Lo, Infectious defaults, Quantitative Finance 1, p. 382-387 (1999); Modelling default correlation in bond portfolios in C. Alexander (Ed.) Mastering Risk, Volume 2: Applications Financial Times, Prentice Hall, p. 141 - 151 (2000).

[3] R. Jarrow and F. Yu, Counterparty risk and the pricing of defaultable securities, Journal of Finance 56, p. 1765 (2001).

[4] E. Rogge and Ph. Schönbucher, Modelling dynamic portfolio credit risk, Working Paper, Imperial College, London, ABN Amro Bank, London, and ETH, Zürich (February 2003).

[5] R. Frey and Jochen Backhaus, Interacting defaults and counterparty risk; a Markovian approach, Working Paper, University of Leipzig (July 2003).

[6] K. Giesecke and St. Weber, Credit contagion and aggregate losses, Working Paper, HumboldtUniversität zu Berlin and TU Berlin (September 2002); Cyclical coorelation, credit contagion and portfolio losses, to appear in Journal of Banking and Finance (January 2003).

[7] K. Giesecke and St. Weber, Cyclical coorelation, credit contagion and portfolio losses, to appear in Journal of Banking and Finance (January 2003).

[8] D. Egloff, M. Leippold, and P. Vanini, A simple model of credit contagion, Working Paper,University of Southern Switzerland (June 2003).

[9] S. Keenan, Historical default rates of corporate bond issues, 1920-2000, Moody's Investor Services (2000).

[10] R. Jarrow and S. Turnbull, Pricing derivatives on financial securities subject to credit risk, Journal of Finance, Vol. 50, p. 53-85 (1995).

[11] D. Lando, On Cox processes and credit risky securities, Review of Derivatives Research, Vol. 2, p. 99-120 (1998).

[12] D. Duffie and K. Singleton, Modeling term structures of defaultable bonds, Review of Financial Studies, Vol. 12, p. 687-720 (1999).

[13] R. Merton, On the pricing of corporate debt:tThe risk structure of interest rates, Journal of Finance, Vol. 29, p. 449-470 (1974).

[14] R. Geske, The valuation of corporate liabilites as compound options, Journal of Finance and Quantitative Analysis, Vol. 12, p. 541-552 (1979).

[15] Ian Iscoe, Alex Kreinin and Dan Rosen, An integrated market and credit risk portfolio model, Algorithmics Research Quarterly, Vol. 2 (3), p. 21-37 (September 1999).

[16] F. Yu, Default correlation in reduced-form models, Working Paper, University of California, Irvine (2002). 
[17] L.E. Reichl, A modern course in statistical physics, Wiley-VCH (1998).

[18] M. Scheffer, S. Carpenter, J. A. Foley, C. Folke, and B. Walker, Catastrophic shifts in ecosystems, Nature, 413591 (2001).

[19] R. Kühn and P. Neu, Functional correlation approach to operational risk in banking organizations, Physica A 322, p. 650 (2003).

[20] M. Leippold, and P. Vanini, The quantification of operational risk, Working Paper,University of Southern Switzerland (November 2003).

[21] Basel Committee on Banking Supervision, The New Basel Accord, $3^{\text {rd }}$ Consultive Document, URL: http://www.bis.org, Basel (May 2003).

[22] M. B. Gordy, A comparative anatomy of credit risk models, Journal of Banking and Finance, Vol. 24, p. 119-149 (2000).

[23] M. B. Gordy, A Risk-Factor Model Foundation for Ratings-Based Capital Rules, Journal of Financial Intermediation, Vol. 12(3), p. 199-232 (2002).

[24] J.P. Morgan Global Research, CreditMetrics ${ }^{\mathrm{TM}}$ : The Benchmark for Understanding Credit Risk, Technical Document, URL: www.creditmetrics.com, New York (1997).

[25] S. Kealhofer, Portfolio Management of Default Risk, Net Exposure, Vol. 1 (2), (1998); KMV Corporation, Credit Monitor ${ }^{\mathrm{TM}}$ and Portfolio Manager ${ }^{\mathrm{TM}}$, New York, (1996), URL: www.KMV.com; O. A. Vasicek, The loan loss distribution, Technical Report, KMV Corporation (1997).

[26] R. L. Mc Greevy, Reverse Monte Carlo modelling, J. Phys C 13, R877-R913 (2001) 